\title{
Modeling the Product Development Process as a Dynamic System with Feedback
}

\author{
Ying-Kui Gu${ }^{1}$, Jung Y. Choi ${ }^{2}$, and Hong-Zhong Huang ${ }^{3}$ \\ 'School of Mechanical and Electronical Engineering, Jiangxi University \\ of Science and Technology, Ganzhou, Jiangxi, 341000, P.R. China \\ ${ }^{2}$ Department of Information Systems \& Operations Management, Raj \\ Soin College of Business, Wright State University, 3640 Colonel Glenn \\ Highway, Dayton, Ohio 45435 USA jung.choi@wright.edu \\ ${ }^{3}$ School of Mechatronics Engineering, University of Electronic Science \\ and Technology of China, Chengdu, Sichuan, 610054, P.R. China
}

\begin{abstract}
Effective product development process modeling can help manage the overall process efficiently and organize multi-functional team to develop product concurrently and cooperatively. In this paper, the development process is viewed as a dynamic system with feedback on the basis of feedback control theory. The dynamic model and its design structure matrix are developed. The model and its design structure matrix can be divided farther to reflect the interaction and feedback of design information. The mode and direction of the development process can be selected to satisfy constraints of process data flow and process control. A fuzzy evaluation method is presented to evaluate the performance of the dynamic development process; this allows the development process to be optimized based on re-organizing design constraints, reorganizing design processes and re-organizing designer's preferences. An application shows that modeling the product development process as a dynamic system with feedback is a very effective method for realizing life cycle design, optimizing the whole development process, improving the degree of concurrent, speeding information flow and reducing modification frequency.
\end{abstract}

\section{Introduction}

The demand for higher quality and lower cost products with shorter development lead-time to meet the dynamic global market's needs has made the performance of 
product development projects an increasingly important area of competitive advantage. In response to these challenges many industries have shifted from a sequential, functional development paradigm to a concurrent, team based paradigm. Increasing concurrence and cross functional development also dramatically increases the dynamic complexity of product development [1-3].

Effective modeling of product development can optimize the development process and the accumulation of design information, and it also can improve the degree of concurrent, optimize the design structure, improve product quality, and cut development cost and time. However, in practice the development process has dynamic uncertainty, time overlapping and constraint coupling, which coupled with the imperfection and fuzziness of design information leads to fluctuations in resource allocation and the accumulation of information that hampers process [3].

Moreover, product development itself is a complex, dynamic and uncertain system with feedback, and rather resembles a network (process net), where processes are highly interconnected, including feedback-loops and interactions on different hierarchical levels [4]. Because the feedback control system and product development process share the common feature of information feedback, feedbackcontrol theory can be viewed as a very effective method for modeling the product development process.

This paper aims to analyze the dynamic characteristics of the product development process, model the product development process as a dynamic system with feedback, and present a suitable method to evaluate the performance of product development process. Section 2 gives a dynamic model with feedback and a design structure matrix for the product development process. Section 3 provides process evaluation and Section 4 illustrates a case of applying the model to Gear Transmission System Development. The research results are discussed in Section 5. Finally, conclusions are provided in Section 6.

\section{A Dynamic Model of the Product Development Process with Feedback}

\subsection{Feedback Control Theory}

Feedback control theory arose from the need to analyze the stability and performance of technological systems by means of information interaction and feedback. It, then, can be used as a very effective tool to model the dynamic product development process.

There are two methods for improving the feedback process. One is to simplify the process and delete the redundant processes that have no value to system output. The other is to strengthen the information feedback and process control.

In order to change a system output as expected by designers, all states in the system should be well controlled. There are two necessary and sufficient conditions that should be satisfied.

(i) The system output can affect all the states in the system. 
(ii) All information in the system should be obtained.

In order to eliminate the adverse influence of process feedback, information interactive feedback should be strengthened to optimize the development process. In the development process, both the positive feedback and negative feedback processes should be strengthened to make the balance point of the development process either be transferred or be perfected in the initial point. In this way the design resources and process programming can be optimized.

\subsection{Dynamic Characteristics of the Product Development Process}

Many uncertain factors bring disturbance to the development system to some extent, and make the system unstable, which can stop or suspend the development process. The instability of a development process will be resulted in poor quality, delay in development time and high development cost.

In order to reduce the instability, the product development should be viewed as a dynamic system with feedback. A dynamic system has dynamic characteristics in each development process where the process has three different states: proceeding state, stop state and feedback state (see Figure 1).

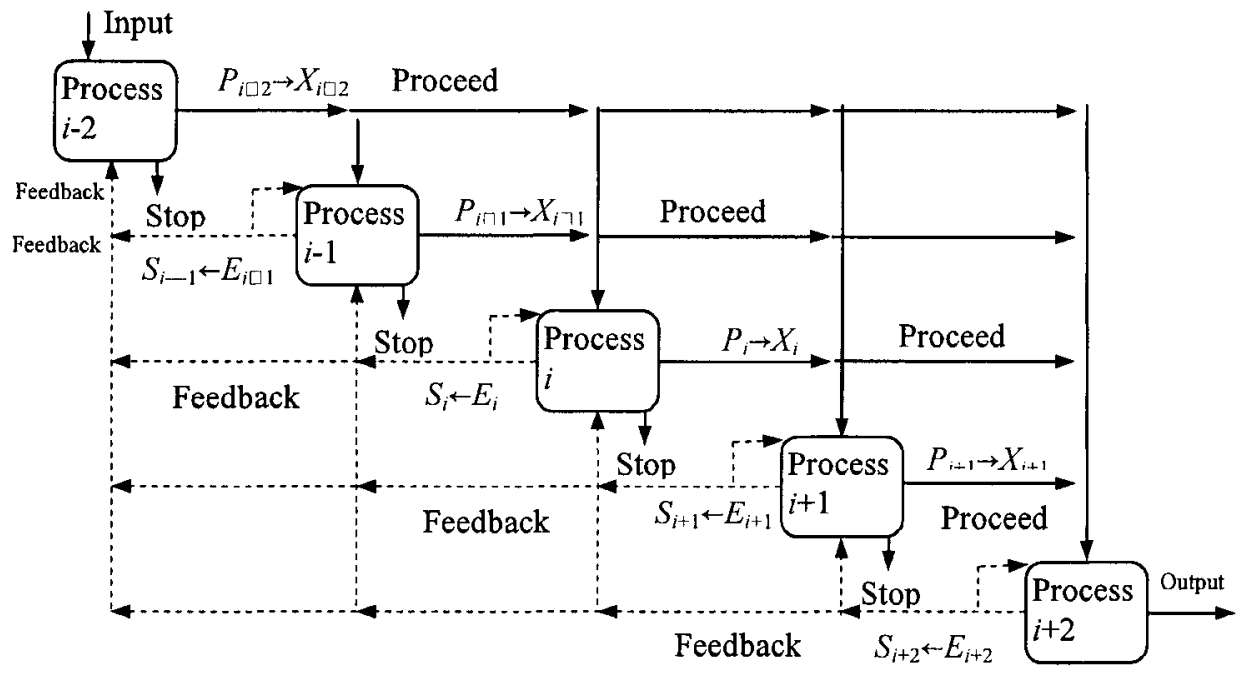

Fig. 1. Dynamic model of development process

In Figure 1, the model can be seen as two parts by the diagonal. The upper right part describes the proceeding state and the lower left part describes the feedback state. The black points in the figure express constraint evaluation points. For 
instance, the satisfaction of a constraint evaluation point determines the proceeding modes of development process (i.e. serial mode and parallel mode) and process flow. However, it should be pointed out that the satisfaction of the constraint evaluation point is only the necessary condition to decide process flow, not the sufficient condition. Based on the satisfaction degree and satisfaction number to constraint evaluation points, many process flows could be selected.

Due to the time characteristic of product development, there exist several time relations between processes on the basis of the satisfaction degree, satisfaction number and satisfaction sequence to constraint evaluation points.

However, meeting the constraint evaluation points is only the necessary condition, not the sufficient condition to decide time series of a process. If the relations above want to be followed strictly, the preconditions and transform conditions of a process, i.e. process control flow, must be satisfied. The feedback state of a process can be depicted by lower left part of Figure 1. Let $X=\left(X_{i-2}, X_{i-1}, X_{i}, X_{i+1}, X_{i+2}\right)$ be the state vector of the dynamic system. The state vector not only could be the state that the designer masters the customer's requirements or the customers understand and express their requirements, or the state that the designer masters the design technology related to the product in the design process, but also could be the manufacturing state in the development process, or the state that the product satisfies the customer's requirements after it was checked and tried out. The state vector has a time characteristic, and its dimension depends on the complexity of the system. Moreover, the state vector is a fuzzy concept. The ideal state can be expressed as 1 , and the most ambiguous state can be expressed as 0 .

Let $P=\left(P_{i-2}, P_{i-1}, P_{i}, P_{i+1}, P_{i+2}\right)$ be the set of state vectors of the dynamic system.

$$
P_{k}: \quad X_{k-1} \times \prod_{n=k}^{i+2} S_{n} \rightarrow X_{k}, k=i-2, i-1, i, i+1, i+2
$$
evaluated.

Let $E=\left(E_{i-2}, E_{i-1}, E_{i}, E_{i+1}, E_{i+2}\right)$ be the feedback state after the process is

$$
E_{k}: \quad X_{k} \rightarrow S_{k}, k=i-2, i-1, i, i+1, i+2
$$

State feedback is the precondition that makes the development process be carried out successfully. When the process proceeds, the process information should be fed back to all the upstream processes. From the viewpoint of control theory, this is a full-feedback model. Information feedback can make the constraints be adjusted in advance and make design information self-supplement, which can eliminate the influence of system perturbation and make the development process collaborative and optimal.

Theoretically speaking, full-feedback of information not only benefits the process collaboration and information interaction, but also makes the design information be fed back completely and embodies the dynamic characteristic adequately. However, under the full-feedback state, the more feedback loops there are, and the more the difficult the operation is. Moreover, it can result in redundant feedback of design information. Redundant feedback is a main reason that can make 
system unstable and result in the bad convergence of system optimization. Therefore, in order to increase the maneuverability of process programming, and to adapt the requirements of multi-function team and a multi-disciplinary working mode [5], not only should the number of feedback loops be reduced, but also the correct design information should be fed back to the correct location in the right time, and the redundant feedback of design information should be reduced. Therefore, the dynamic model proposed above should be modified to reduce intermediate feedback loops and make all the design information be fed back to the initial design stage. Through the revised dynamic model, the designers can analyze the problems together, and find where the problems occur. The model can be described as shown in Figure 2.

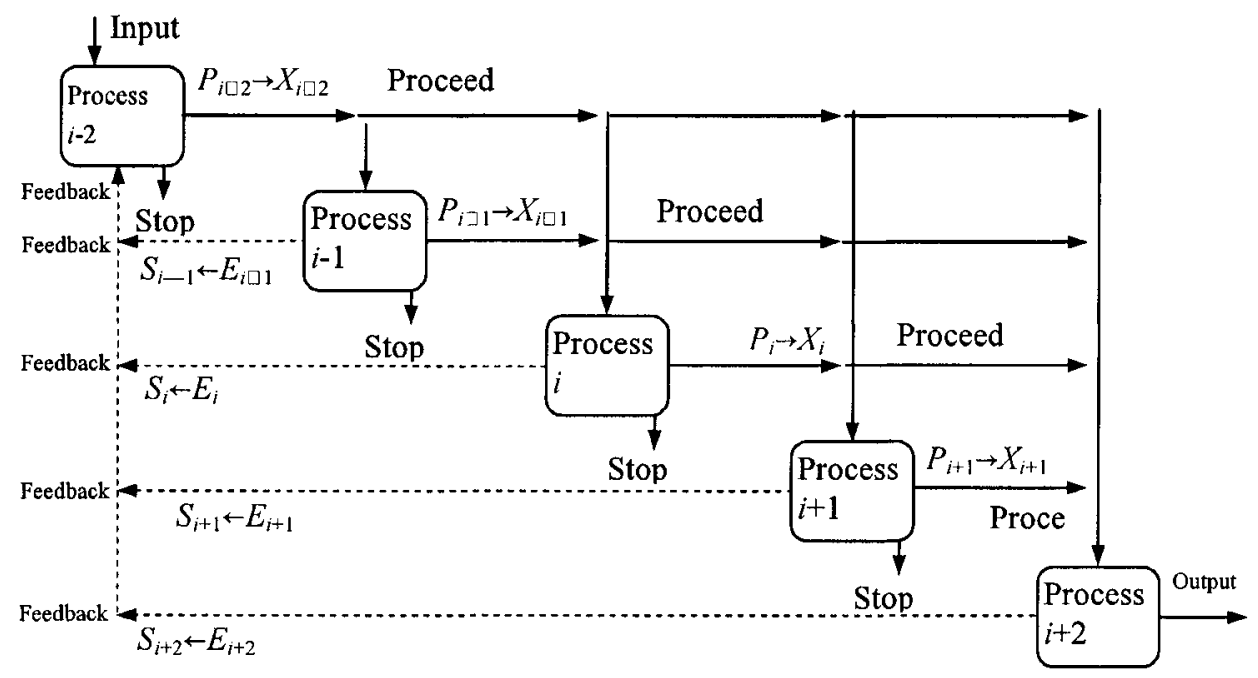

Fig. 2. Dynamic model of revised development process

After the process model is revised, the state mapping of system can be denoted by

$$
\begin{aligned}
& P_{k-2}: \quad X_{k-1} \times \prod_{n=k}^{i+2} S_{n} \rightarrow X_{k}, k=i-2 \\
& P_{k}: \quad X_{k-1} \rightarrow X_{k}, k=i-1, i, i+1, i+2
\end{aligned}
$$

The state feedback of the system can be expressed as

$$
E_{k}: \quad X_{k} \rightarrow S_{k}, k=i-2, i-1, i, i+1, i+2
$$


Design Structure Metrics (DSM) provides a compact and clear representation of a complex system and a capture method for the interactions/interdependencies/interfaces between system elements (i.e. sub-systems and modules) [6-9]. The DSM method assumes that each design task can be modeled as an information processing task, using and creating information. The output information from one task becomes the input information to another task. The input/output relationships may include cycles, which indicate the need for iteration. Tasks in the matrix may be re-sequenced [1].

In this paper, a new design structure matrix is defined and used to describe the dynamic development process with feedback. The design structure matrix can be divided into two parts by the diagonal. The one part describes the interdependence relations among processes, i.e. the correlative information among different processes. The information can be the number of parameters of a process output or the communication quantity among processes. The other part describes the feedback relations among different processes, and the feedback information can be the number of feedback loops or the number of feedback parameters.

\section{Process Evaluation}

Process evaluation is based on factors such as quality, cost and development time. Let $\left\{u_{1}, u_{2}, \cdots, u_{n}\right\}$ be vector set of evaluation factors, with the range of value representing the degree of membership of the corresponding factor's satisfaction degree. Let $\mu_{i}\left(v_{i}\right)$ be the degree of membership, and $v_{i}$ be the corresponding value of $u_{i}$. The weight vector of evaluation factors can be given by experts and expressed as $\left\{w_{1}, w_{2}, \cdots, w_{n}\right\}$.

The evaluation method can be carried out step by step as follows.

(i) If $v_{i} \notin \mu_{i}\left(v_{i}\right)$, then the feedback information is

- The corresponding indices of structure matrix.

- The difference between the index and its lowest value.

(ii) If $v_{i} \in \mu_{i}\left(v_{i}\right)$, giving the threshold of satisfaction degree and denoted by $S_{m}$. Let $\mu_{i}\left(v_{i}\right)$ be the degree of membership of the evaluation factor. Such that

$$
S=\sum w_{i} \mu_{i}\left(v_{i}\right)
$$

- $\quad$ If $S \geq S_{m}$, the process is ${ }^{i}$ sdtisfied.

- If $S<S_{m}$, it has to carry out the process optimization and information feedback. The optimization model is as follows:

$$
\operatorname{Min} A-A^{\prime} \|_{1}^{1} ; \text { S.t } \sum_{\max } w_{i} \mu_{i}\left(v_{i}\right) \geq S_{m} ; \mu_{v}^{\min } \leq \mu_{i}\left(v_{i}\right) \leq 1
$$

where $A=w \mu(v) . A^{\prime}=w \mu_{m=1} \cdot \mu^{\max }$ is the biggest vector of degree of membership. $\mu_{v}^{\min }$ is the least vector of degree of membership. $\left\|A-A^{\prime}\right\|_{\mathrm{P}}^{\mathrm{P}}$ is the norm that $\mathrm{P}>1$. 
The feedback information $\Delta$ is:

(a) If $v_{i \text { lower limit }}>v_{i}$, then $\Delta_{i}=\left|v_{i \text { lower limit }}-v_{i}\right|$

$$
\begin{aligned}
& \text { If } v_{i \text { lower limit }}<v_{i}<v_{i \text { upper limit }} \text {, then } \Delta_{i}=\mid v_{i} \text { upper limit } \\
& \text { If } v_{i \text { upper limit }}<v_{i} \mid \\
& \text { (b) then } \Delta_{i}=\left|v_{i}-v_{i \text { upper limit }}\right|
\end{aligned}
$$

After the feedback information is fed back to the initial design stage, the multifunction design team can carry out three re-organization activities as follows based on the practical development environment to optimize the development process: (1) re-organize constraints, (2) re-organize the process, and (3) re-organize the structure of the designer's preference.

\section{Application to Gear Transmission System Development}

The development of a gear transmission system for the ZL50G loader is an example analysis of the dynamic characteristics of product development. In order to develop a high quality product in short time, the dynamic characteristics of the development process should be analyzed to establish an effectual process model. At the same time, because the development of a new loader inherits the features of ZL50, the interdependence and feedback relationships are more complicated.

There are seven sub-processes: (1) Prepare product specifications, (2) Preliminary design, (3) Evaluate development cost, (4) Mechanical analysis, (5) Design process features, (6) Analyze the product data of identical series products, and (7) Finalize design details.

From the analysis we identified the following:

(i) Through the dynamic analysis of the development process, the uncertain factors and some rules that can be followed can be found. Therefore, the development process activities can be organized and managed more effectively.

(ii) The development process can be optimized by the optimization of the interdependence and feedback relationships among processes. The number of feedback loops and the complexity of development process are reduced. The number of feedback loops is

$$
b=\sum \sum M_{b}(i, j)=6
$$

(iii) From the analysis of lstructure matrix we can see that the feedback loops exist mainly in processes $2,4,5$ and 6 . That is to say the feedback loops exist mainly in the detailed design stage. By substituting the big feedback cycle with microcycles, the development efficiency can be improved and the redundant feedback can be reduced.

(iv) By improving and optimizing the relations among processes, the degree of concurrent can be improved and the development time can be reduced. Most process can be carried out in parallel or partly in parallel. 
We select cost, weight and quality of transmission as evaluation factors; they can be denoted by an evaluation vector, $U=(\mathrm{u} 1, \mathrm{u} 2, \mathrm{u} 3)=($ cost, weight, transmission quality). The weights of evaluation factors can be given by experts and expressed as a weight vector $W=\left(w_{1}, w_{2}, w_{3}\right)=(0.3,0.3,0.4)$. For a certain process, the value of range and the degree of membership of the corresponding range's satisfaction degree can be given in advance, and the threshold of satisfaction degree $S_{m}$ can also be given in advance. $S_{m}=0.90$. Based on the evaluation results, we can change or adjust the helical angle, material, tooth number, modulus, tooth length, and so on, and this information is fed back to the frontward processes to modify the design processes and design constraints. The final evaluation result is that $S=0.947$. The implementation of dynamic analysis brings great benefits to our development. The development time is shortened more than 30 percent, and the development cost is cut down more than 40 percent.

\section{Discussion}

There exists over-amplitude in product development process, i.e. an iterative process. The number of feedback loops, time of feedback and location of feedback has a very important influence on information interaction and product development. In order to make the development process continue successfully, the correct design information must be fed back to the correct location at the right time, so that the redundant feedback processes can de reduced. Take the state variables in the development process of gear transmission system of ZL50G loader as example, let $X_{1}$ be the state vector that the designer masters the customer's requirements or the customers understand and express their requirements. Let $X_{2}$ be the state vector that the designer masters the design technology related to the product in the design process. Let $X_{3}$ be the state vector that the manufacturing state in the development. Let $X_{4}$ be the state vector that the product satisfies the customer's requirements after it was checked and tried out. As shown in Figure 3, 3(a) denotes that manufacturing process successes after many repetitions, and $3(\mathrm{~b})$ denotes that manufacturing process successes with no repetitions.

The reasonable analysis of dynamic characteristics can bring many additive benefits, such as shortening development time, cutting development cost, improving product quality, and so on. Failure to complete such a reasonable analysis leads to additive losses in the process. The evaluation precision, feedback information and reorganization activities significantly influence the convergence speed of process optimization.

From Figure 3 we can see that the customer's requirements and the instability of design technology are the main contributors to the instability of the development system. Because of increasing new requirements and the emergence of new technology, these two state variables should be emphasized to reduce the overamplitude of the product development process. 
Enhancing the evaluation precision and using the intelligent tools (such as a neural network) to real-time evaluate development process can make the best of the design information and reduce the redundant interactive process.

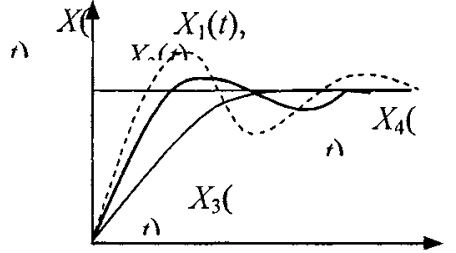

(a) Manufacturing process successes after manv repetitions

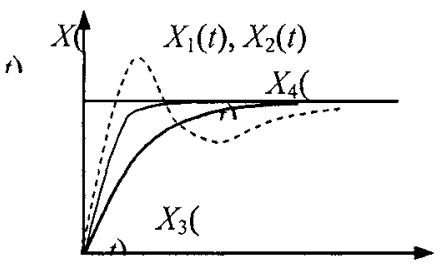

(b) Manufacturing process successes with no repetitions

Fig. 3. The variation of process state variables

\section{Conclusions}

Product development process modeling is the heart of product development activities that determine the engineering productivity and industrial competitiveness. The rapid changing information technology and the competitive nature of global market make the research and application efforts in development process modeling a fast moving target. Modeling the product development process as a dynamic system with feedback is a very effective method for realizing life cycle design, optimizing the whole development process, improving the degree of concurrent, capturing designer's preferences, speeding information flow and reducing modification frequency.

However, due to the complexity of the product development, there are several problems should be taken into account in the future research.

(i) Because some processes are carried out partly in parallel, there exist limitations in the design structure matrix that is expressed as 0 and 1 .

(ii) In order to speed up the convergence of process optimization, the re-organization activities of process, constraints and preference's structure should be implemented in parallel based on the feedback information. 
(iii) The insufficiency of process dynamic analysis and the impropriety of process evaluation and information feedback can increase the instability of system to a great extent.

\section{References}

1. R.P. Smith and J.A. Morrow, Product development processes modeling, Design Study 20(3), 237-261 (1999).

2. D.N. Ford, and J.D. Sterman, Dynamic modeling of product development process. 1997.

3. L.W. Xie, Y.D. Cheng, and G.N. Qi, Evaluation of complexity and preference of integrated product development process management, Journal of Chinese Mechanical Engineering 36(10), 37-41 (2000).

4. H. Negele, et al., Modeling of integrated product development processes (1989).

5. U. Belhe and A. Kusiak, Modeling relationships among design activities. ASME, Journal of Mechanical Design 118(12), 454-460 (1996).

6. D.V. Steward, The design structure system: a method for managing the design of complex systems, IEEE Transactions on Engineering Management 28(3), 71-74 (1981).

7. S.D. Eppinger, et al., A model-based method for organizing tasks in product development Research in Engineering Design 6(1), 1-13 (1994).

8. A. Kusiak and J. Wang, Decomposition of the design process, Journal of Mechanical Design 115, 687-695 (1993).

9. S.D. Eppinger, Model-based approaches to managing concurrent engineering. Journal of Engineering Design 2(4), 283-290 (1991). 